\title{
Observations of equatorial plasma bubbles using a low-cost 630.0-nm all-sky imager in Ishigaki Island, Japan
}

\author{
Keisuke Hosokawa ${ }^{1 *}$ (D) , Kohei Takami ${ }^{1}$, Susumu Saito ${ }^{2}$, Yasunobu Ogawa ${ }^{3}$, Yuichi Otsuka ${ }^{4}$, Kazuo Shiokawa ${ }^{4}$ \\ Chia-Hung Chen ${ }^{5}$ and Chien-Hung Lin ${ }^{5}$
}

\begin{abstract}
Here, we introduce a low-cost airglow imaging system developed for observing plasma bubble signatures in 630.0$\mathrm{nm}$ airglow emission from the $\mathrm{F}$ region of the ionosphere. The system is composed of a small camera, optical filter, and fish-eye lens, and is operated using free software that automatically records video from the camera. A pilot system was deployed in Ishigaki Island in the southern part of Japan (Lat 24.4, Lon 124.4, Mlat 19.6) and was operated for 1.5 years from 2014 to 2016 corresponding to the recent solar maximum period. The pilot observations demonstrated that it was difficult to identify the plasma bubble signature in the raw image captured every $4 \mathrm{~s}$. However, the quality of the image could be improved by reducing the random noise of instrumental origin through an integration of 30 consecutive raw images obtained in $2 \mathrm{~min}$ and further by subtracting the 1 -h averaged background image. We compared the deviation images to those from a co-existing airglow imager of OMTIs, which is equipped with a backilluminated cooled CCD camera with a high quantum efficiency of $\sim 90 \%$. It was confirmed that the low-cost airglow imager is capable of imaging the spatial structure of plasma bubbles, including their bifurcating traces. The results of these pilot observations in Ishigaki Island will allow us to distribute the low-cost imager in a wide area and construct a network for monitoring plasma bubbles and their space weather impacts on satellite navigation systems.
\end{abstract}

Keywords: Ionosphere, Plasma bubble, Airglow, Optical observations, Satellite navigation

\section{Background}

Equatorial plasma bubbles (EPBs) are localized regions in the nighttime equatorial $\mathrm{F}$ region of the ionosphere where the plasma density is significantly depleted below the background level (see a review by Kil 2015 and references therein). They are formed near the magnetic equator by the uplift of low-density plasma from the lower $\mathrm{F}$ region through the Rayleigh-Taylor-type interchange instability (Woodman and Lahoz 1976). Such densitydepleted regions extend along the magnetic field line; thus, EPBs developing toward higher altitude can be observed in the low-latitude region $10-20^{\circ}$ away from the

\footnotetext{
*Correspondence: keisuke.hosokawa@uec.ac.jp

${ }^{1}$ University of Electro-Communications, Chofugaoka 1-5-1, Chofu, Tokyo

182-8585, Japan

Full list of author information is available at the end of the article
}

magnetic equator (i.e., in the region of equatorial anomaly). EPBs are known to introduce a positioning error in the Global Navigation Satellite System (GNSS) such as the Global Positioning System (GPS). This is because the strong depletion of electron density within EPBs modifies the phase and group velocities of the navigation signal traveling through the ionosphere. EPBs have also been recognized as one of the primary sources of GNSS scintillations (e.g., Aaron 1993). That is, the amplitude of the navigation signal received on the ground that has propagated through EPBs often fluctuates. This is primarily due to an interference of radio signals diffracted by plasma density irregularities formed within EPBs (Franke et al. 1984).

As summarized above, the impact of EPBs on the GNSS is clear, indicating the relevance of EPBs in the 
framework of ionospheric space weather. It is especially desirable to understand the triggering process of EPBs for predicting the space weather effects of EPBs. For this purpose, in the last decade, several numerical simulations have been carried out (see a review by Yokoyama 2017), and it has been suggested that the modulation of electron density in the lower ionosphere, such as large-scale wave structures (e.g., Tsunoda 2015 and references therein) or modulation by upward-propagating gravity waves (e.g., Huang and Kelley 1996), is one plausible factor controlling the generation of EPBs. Carter et al. (2014) demonstrated a capability of predicting the occurrence of EPBs by calculating the linear growth rate of the $\mathrm{R}-\mathrm{T}$ instability using the Thermosphere-Ionosphere Electrodynamics General Circulation Model (TIEGCM). More recent study by Shinagawa et al. (2018) succeeded in reproducing the seasonal variation of EPBs by employing a whole atmosphere-ionosphere coupled model GAIA (Groundto-topside model of Atmosphere and Ionosphere for Aeronomy). However, the day-to-day variabilities in the occurrence of EPBs and its impact on the GNSS are still unpredictable. This implies that we need continuous monitoring of EPBs to mitigate the GNSS positioning error.

Several radio techniques have been used to observe EPBs since the 1960s, such as ionosondes (e.g., Maruyama and Matuura 1984) and radar (Woodman and Lahoz 1976). For instance, the ionosonde has been used to observe EPBs as a spread of the F-region trace, which is called spread-F (e.g., Fejer and Kelley 1980). All-sky measurement of the $630.0 \mathrm{~nm}$ airglow intensity, which is proportional to the electron density in the $\mathrm{F}$ region of the ionosphere (Sobral et al. 1993), has also been utilized to visualize the 2D structure of EPBs (Weber et al. 1978). Since the late-1990s, the use of all-sky imagers (ASIs) equipped with a cooled CCD camera has enabled us to detect faint oxygen emission from the F-region altitude with a good signal-to-noise $(\mathrm{S} / \mathrm{N})$ ratio, because backilluminated cooled CCD cameras have a high quantum efficiency of $\sim 90 \%$ for incident photons in visible wavelengths (e.g., Shiokawa et al. 1999, 2009). Using observations of oxygen emission at $630.0 \mathrm{~nm}$, several detailed characteristics of EPBs have recently been discovered, for example, their geomagnetically conjugate appearance between northern and southern hemispheres (Otsuka et al. 2002; Shiokawa et al. 2004).

However, the conventional ASIs, utilizing a cooled CCD detector, tend to be large in the total size and expensive; thus, it has still been difficult to visualize the large-scale structure of EPBs by setting up multiple ASIs at multiple stations and constructing a dense network of airglow observations. To overcome this limitation, we have developed a small and cheap ASI system for observing the 630.0-nm airglow signature of EPBs. Similar small and low-cost ASIs have been deployed and installed into several places in the polar region in both hemispheres (Ogawa et al. 2020). Hosokawa et al. (2019) used one of the ASIs at Longyearbyen in Svalbard, Norway and confirmed its capability of detecting faint airglow signatures of polar cap patches whose airglow intensity is a few hundreds of Rayleigh. As a next step, we carried out a pilot observation at low latitudes to evaluate the feasibility of utilizing the ASIs for monitoring EPBs.

\section{Experimental arrangement of the pilot observations}

For the pilot observation, we developed a low-cost ASI system by assembling a small CCD camera (WAT-910HX of Watec Co. Ltd.), small fish-eye lens (YV2.2X1.4ASA2 of Fujinon Co. Ltd.), and small-aperture optical filter (Edmund Co. Ltd). The diameter of the optical filter is $25 \mathrm{~mm}$. The center wavelength of the bandpass filter is $632 \mathrm{~nm}$, and the FWHM of the bandwidth is $10 \mathrm{~nm}$, which mainly covers the oxygen emission at $630.0 \mathrm{~nm}$. The major advantage of this ASI system is its low cost; the entire cost including a control PC and a video encoding device is approximately 1000 USD, which is roughly $1-2 \%$ of the cost of the conventional cooled CCD ASIs. The other advantage is that the imager is small and simple. Figure 1a shows a picture of the camera, and the size of the imager is roughly $5 \mathrm{~cm} \times 5 \mathrm{~cm} \times 10 \mathrm{~cm}$ (weight is $\sim 245 \mathrm{~g}$ ). The imager is directly connected to a Linux PC laptop (Fig. 1c) through the Video/USB interface (PCA-DAV3: Fig. 1b). The video signal from the camera is digitized on the Linux PC using free video-capturing software called Motion. Motion is a configurable program that can process incoming video signals from cameras and save them as static images automatically (detail of the software can be found in https://motion-proje ct.github.io). During the entire period of the pilot observation, we capture all-sky images every $4 \mathrm{~s}$ as 8 -bit JPEG images, which are, hereafter, called "raw images."

The instrumental details of the low-cost airglow imager are documented in Ogawa et al. (2020) including the description of its calibration and performance. However, we briefly describe such information in this section. Ogawa et al. (2020) carried out a calibration experiment using a 76-in. integrating sphere (Labsphere LMS-760) at National Institute of Polar Research (NIPR) of Japan and confirmed that the low-cost ASI has good linearity (target intensity versus count) even though the data from the camera are saved as compressed JPEG images. The average noise level is $\pm 30 \mathrm{R}$ for the $4.3 \mathrm{~s}$ exposure time in 630.0-nm setting, which is used for the current airglow observations of EPBs. Note that this average noise level was calculated when the background luminosity 


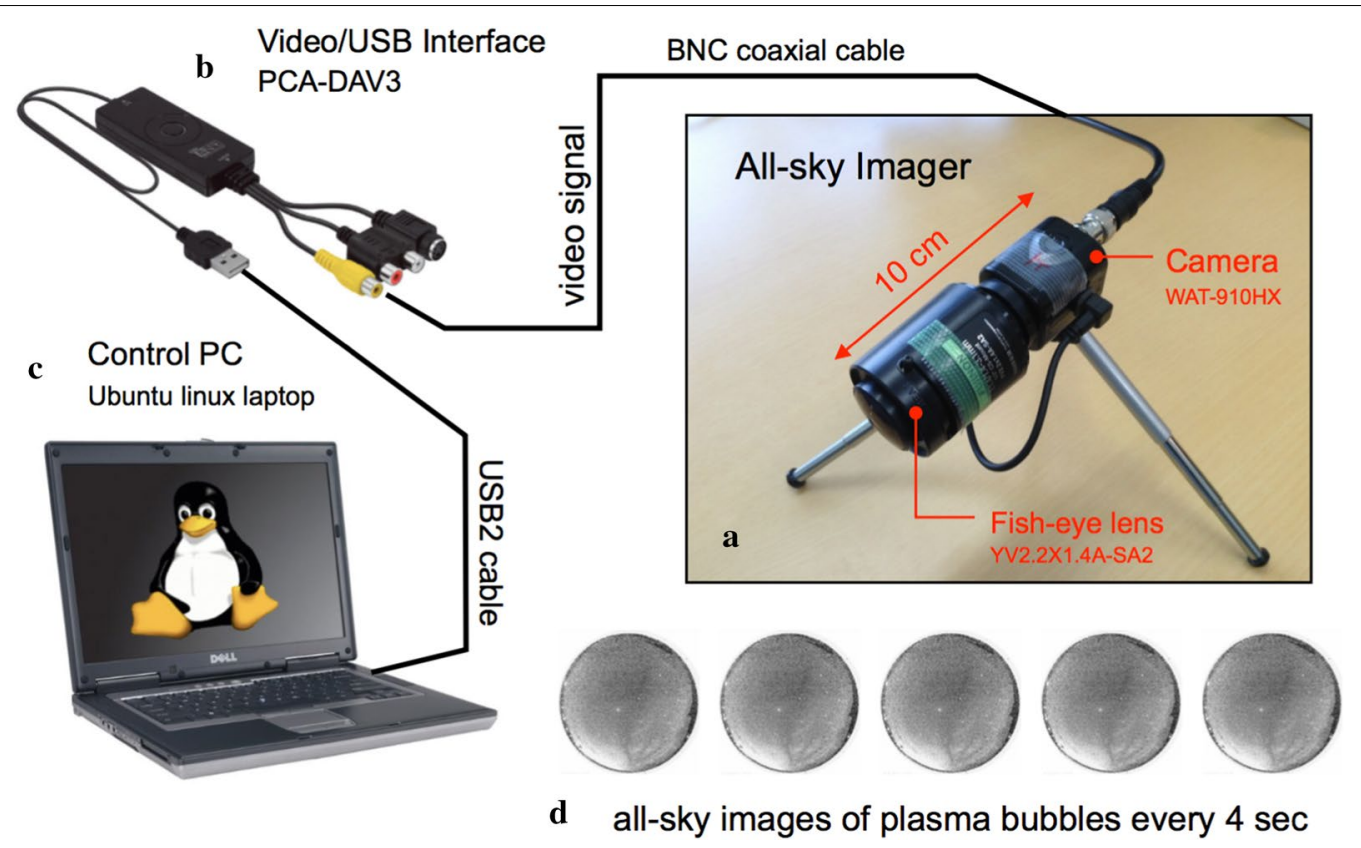

Fig. 1 Schematic representation of low-cost airglow imager system used for the pilot observations in Ishigaki Island, Japan

was $\sim 600 \mathrm{R}$. The dynamic range of the ASI is $20-1200$ $\mathrm{R}$, which is determined by the 8-bit digitalization of raw count. The upper limit of the dynamic range of $1200 \mathrm{R}$ is sufficient for airglow measurements (obviously insufficient for auroral measurements; thus, we needed to shorten the exposure time for observing aurora).

Derivation of the absolute optical intensity in unit of Rayleigh has been done also using the calibration data obtained at NIPR. The procedure is documented in Hosokawa et al. (2019) which employed the same imager for observing polar cap airglow patches in Longyearbyen $\left(78.1^{\circ} \mathrm{N}, 15.5^{\circ} \mathrm{E}\right)$, Norway. When deriving the absolute optical intensity, we estimate the dark count using pixels outside the FOV of the ASI and subtract it from the raw count values. After the subtraction, we convert the raw count to the absolute optical intensity in units of Rayleigh using the sensitivity values (Count $R^{-1} \mathrm{~s}^{-1}$ ) estimated from the calibration data obtained at NIPR. Since we do not observe background continuum emission at other wavelengths in Ishigaki Island $\left(24.4^{\circ} \mathrm{N}, 124.4^{\circ} \mathrm{E}\right)$, we have not subtracted the contribution of background emission. Hence, the derived absolute optical intensity in Rayleigh includes a certain contribution of background continuum emission. However, Hosokawa et al. (2019) pointed out that the offset is $\sim 20 \mathrm{R}$ for the cases of airglow observations in the polar cap region.

To carry out the pilot observation, we installed the lowcost airglow imager into an observation site in Ishigaki Island, Okinawa, Japan. The magnetic latitude of Ishigaki
Island is $19.6^{\circ} \mathrm{N}$, which is close to the poleward edge of the equatorial anomaly. The pilot observation was carried out from August 2014 to the end of April 2016, during which we experienced two major disruptions of measurement in 2015. A conventional cooled CCD imager has also been operative in the same place as part of the OMTI network (Shiokawa et al. 1999, 2009). This allows us to compare the quality of the airglow images from these two systems and evaluate the performance of the low-cost airglow imager.

\section{Pilot observations and image processing}

On the night of Feb 14, 2015, a series of EPBs was observed in Ishigaki for $7 \mathrm{~h}$ from 09 to $19 \mathrm{UT}$ (21 to 28 LT). Figure 2a shows a raw all-sky image from the lowcost imager taken when an EPB was located near the center of the field-of-view. As described in the previous section, the exposure time of this raw image was $4 \mathrm{~s}$, which is much shorter than that used in the ASI of OMTIs (165 s). The equatorward half of the field-of-view was much brighter than that in the poleward half. This is primarily due to the enhanced electron density in the equatorial anomaly located immediately equatorward of Ishigaki Island. The signature of the EPB is seen as a dark, tree-like structure near the center of the image extending poleward from the southern edge of the field-of-view. Although there is an indication of an EPB in the raw image, it is difficult to see their spatial structure in detail because of the limitation in image quality. 

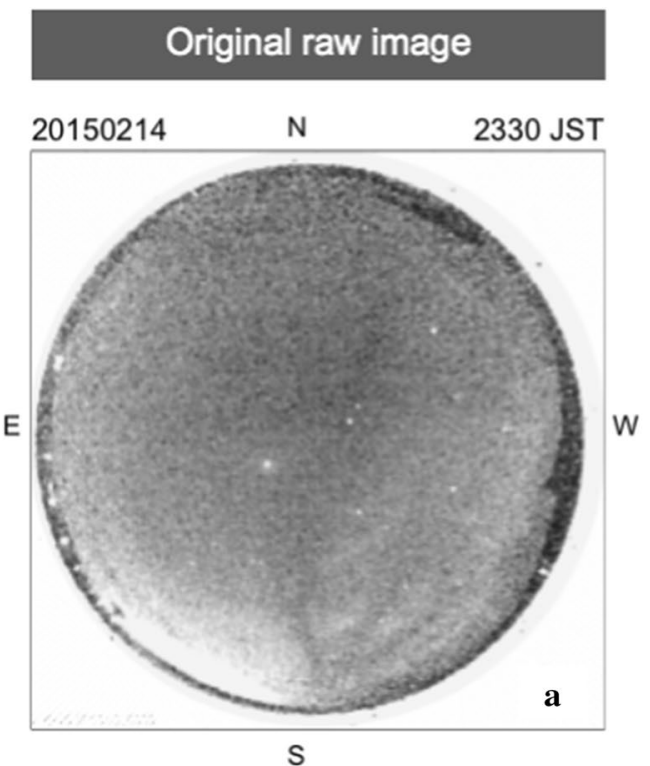

\section{Average of 30 images}

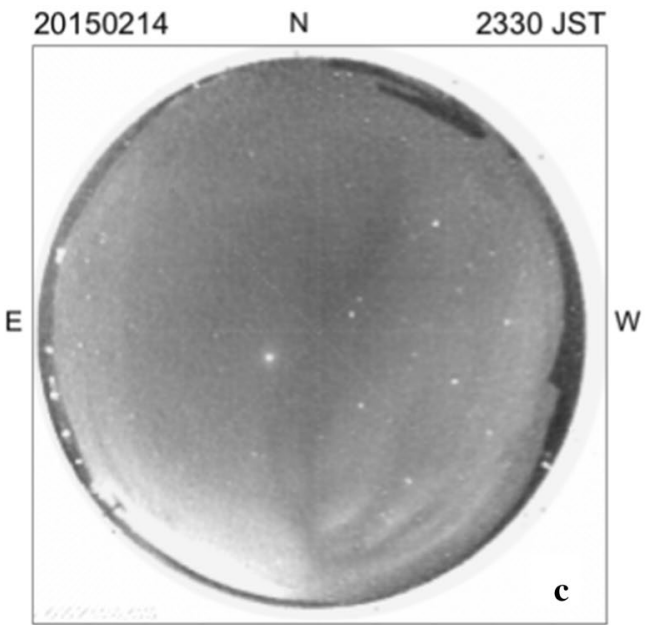

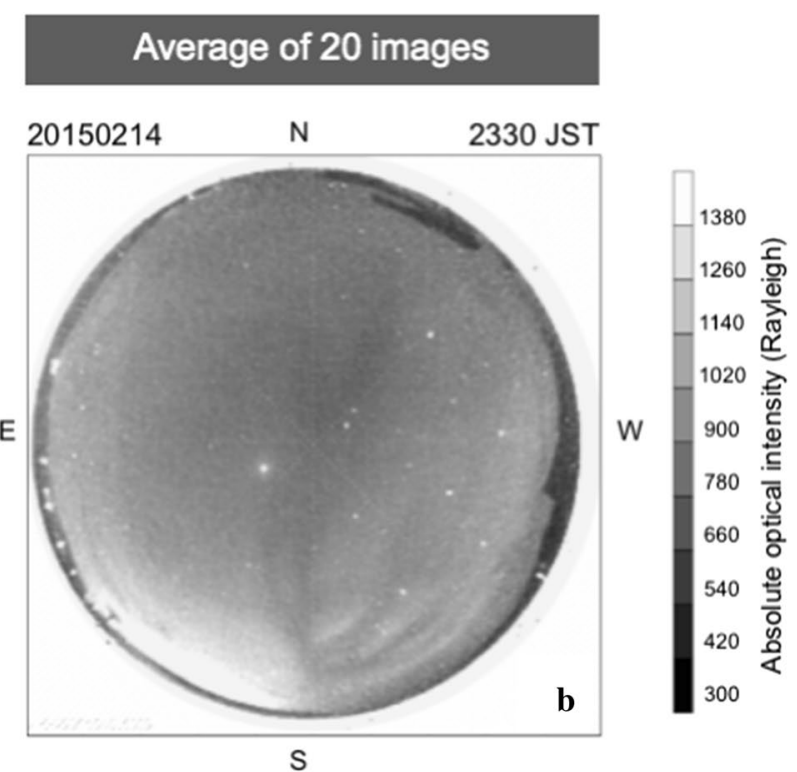

\section{Average of 40 images}

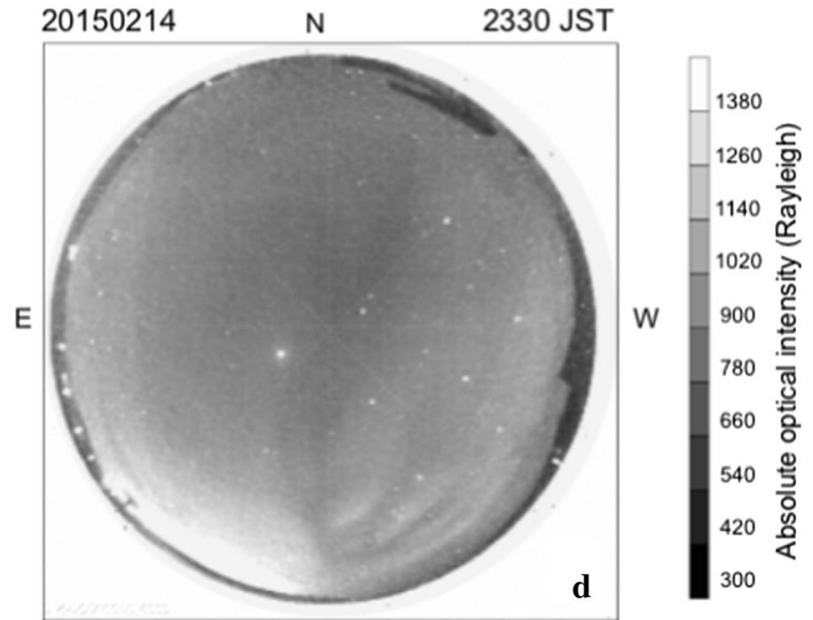

Fig. 2 All-sky 630.0-nm images obtained at 2330 JST on February 14, 2015: a raw all-sky image obtained with an exposure time of 4 s; $\mathbf{b}$ average of 20 consecutive images (total integration time of 80 s); c average of 30 consecutive images (total integration time of 120 s); $\mathbf{d}$ average of 40 consecutive images (total integration time of $160 \mathrm{~s}$ )

One of the problems of the low-cost ASI is the low $\mathrm{S} / \mathrm{N}$ ratio due to the random shot noise of the noncooled CCD camera. To remove such unwanted noise in the raw image, we first investigated how the quality of the data is improved by averaging consecutive raw images. Figure $2 \mathrm{~b}-\mathrm{d}$ respectively show the "average images" derived by integrating 20,30, and 40 consecutive raw images. These respectively correspond to total integration times of 80,120 , and $160 \mathrm{~s}$. It is clearly seen that the contribution of shot noise of instrumental origin is significantly reduced by applying image integration. However, the processed image can be blurred because EPBs move in the zonal direction with a speed of approximately $100 \mathrm{~m} / \mathrm{s}$. Thus, we also need to take this blurring effect into account when we determine the best integration time. An average of the absolute optical intensity in the central part of Fig. 2a is 900 $\mathrm{R}$ and the standard deviation is $80 \mathrm{R}$. The absolute optical intensity decreases by $\sim 40 \mathrm{R}$ within the EPB near the center of the image in Fig. $2 \mathrm{~b}$; thus, the $\mathrm{S} / \mathrm{N}$ ratio is $\sim 0.5$ in this case $(\sim 40 \mathrm{R} / 80 \mathrm{R})$. When we integrate 20,30 , and 40 images, the standard deviation does not 
change so much ( $44 \mathrm{R}, 43 \mathrm{R}$, and $43 \mathrm{R}$, respectively). The standard deviation value does not change so much beyond the integration of 30 images. By comparing both the noise reduction and blurring effect by averaging, the integration of 30 raw images, corresponding to an integration time of $2 \mathrm{~min}$, would be appropriate for observations of plasma bubbles with the low-cost ASI. An attached additional movie file, covering the full sequence of the interval, demonstrates that the spatial structures of EPBs, including their bifurcating traces, are clearly recognized in the average image (see Additional file 1).
Although we are able to identify the bifurcating structures of EPBs in the average image, the signature is still faint. This is particularly because the background count is larger than that of the deviation component associated with EPBs (the background intensity of the original raw image is $\sim 896 \mathrm{R}$ and the optical intensity decreases by $\sim 40 \mathrm{R}$ within the EPB trace near the center of the image). Thus, we applied another image processing to the average image. Figure 3a again shows the 2-min average image, which is the same as that shown in Fig. 2c. Figure $3 \mathrm{~b}$ shows a background image derived by averaging 900 images (duration of this averaging is $1 \mathrm{~h}$ ) obtained
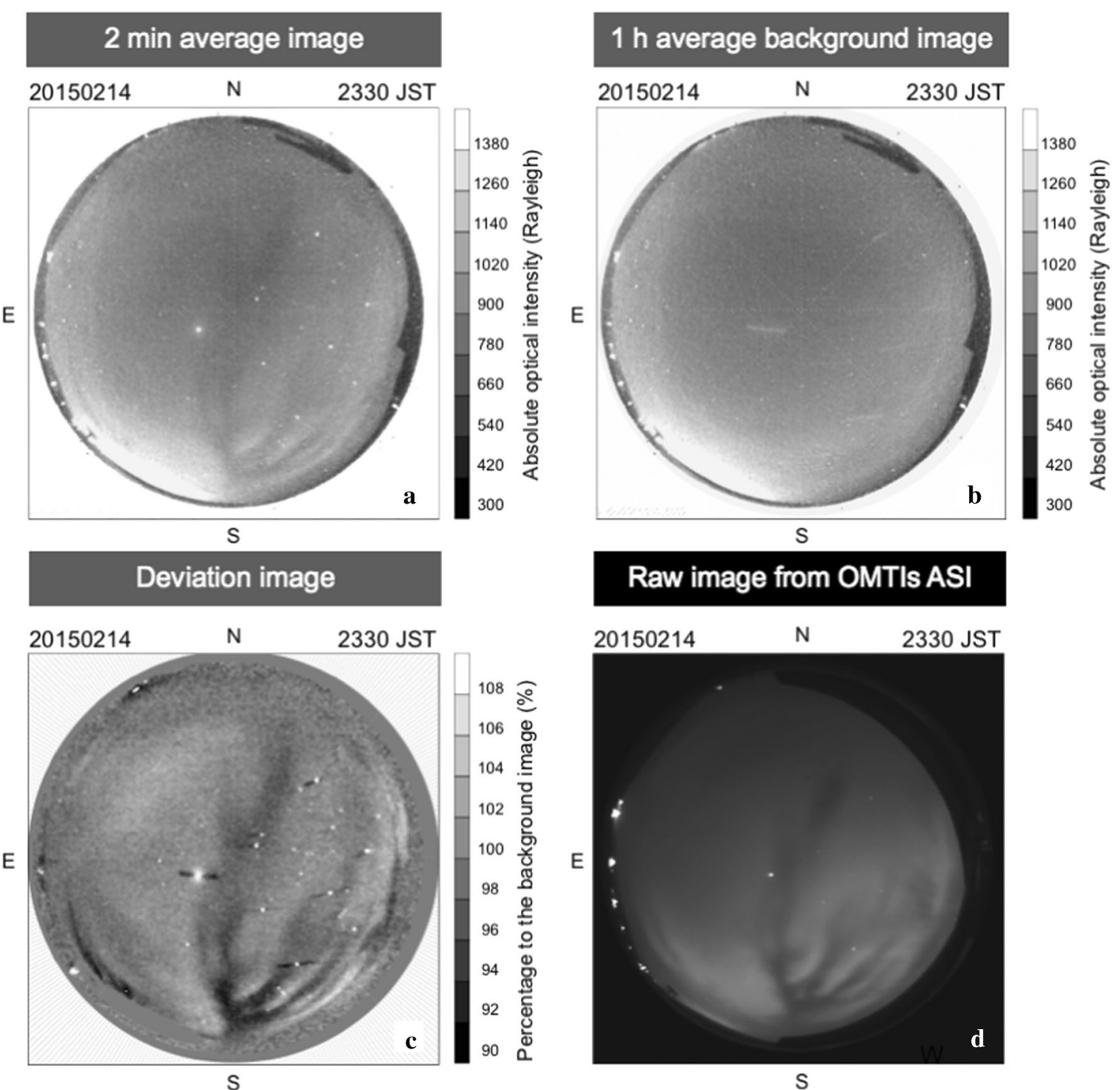

\section{Raw image from OMTIs ASI}

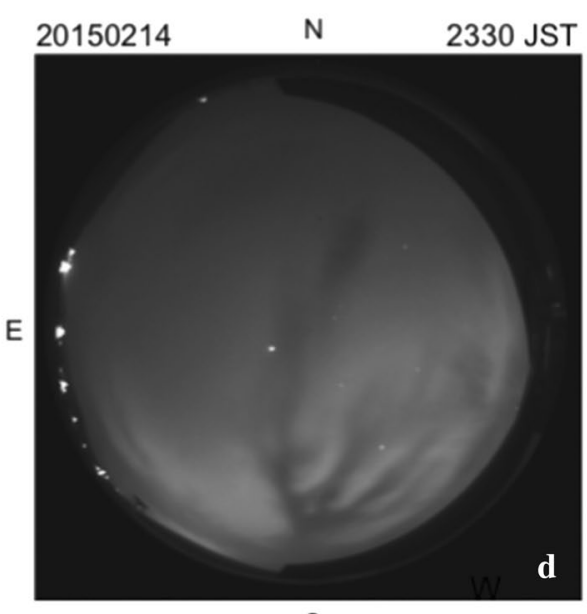

S

Fig. 3 Procedure of image processing: a 2-min average all-sky image (same as Fig. 2c); $\mathbf{b}$ background image obtained by averaging all the images obtained in the surrounding 1-h interval; $\mathbf{c}$ deviation image derived by calculating the "percentage of the 2-min average image (Fig. 3a) to the 1-h background image (Fig. 3b)"; d raw all-sky image from OMTI ASI obtained at the same time in the same place 
in an hour surrounding the time of the average image in Fig. 3a. In the 1-h average image, the large-scale structure of the equatorial anomaly, i.e., prominent enhancement in the southern part of the field-of-view, is noticeable. Figure $3 \mathrm{c}$ shows the so-called deviation image derived by calculating the "percentage of the average image (Fig. 3a) to the 1-h background image (Fig. 3b)". Note that the color scale used here ranges from 90 to $110 \%$, where $90 \%$ means that the optical intensity decreases by $10 \%$ from the background level. In the deviation image, the signature of the EPB is more contrasted and its detailed structure becomes more distinct.

Now, we compare this deviation image to an image obtained by a conventional cooled CCD imager operating in the same place. Figure 3d shows an image of 630.0-nm airglow from the OMTI ASI, which was taken at the same time in the same place. The exposure time of the OMTI ASI was $165 \mathrm{~s}$, which is slightly longer than the exposure time of the 2-min average image of the low-cost ASI. The quality of the deviation image from the low-cost ASI is almost comparable to the raw image from the OMTI ASI, which implies that the low-cost imager can be used, at least, for monitoring the appearance and development of EPBs.

\section{Statistics of occurrence of EPBs}

Figure 4a shows the occurrence distribution of EPBs during the 1.5-year period of pilot observations in Ishigaki. The extraction of EPBs was done by visual inspection. The intervals when at least an EPB was observed within the field-of-view are indicated by the horizontal lines. It is well known that the activity of EPBs is highest in equinoctial months during solar maximum in the Asian longitudinal sector (Kil et al. 2009). The occurrence distribution shown in Fig. 4a is fairly consistent with the results of these previous studies. Namely, the occurrence of EPBs is almost confined in the equinoctial months, which is more clearly seen in the first year of the pilot observation. The occurrence of EPBs is also known to be pronounced in the early evening interval soon after sunset (e.g., Makela et al. 2004). This tendency is also seen in the current statistics, where most EPBs appear immediately after $20 \mathrm{LT}$, and the occurrence is lower in the postmidnight interval.

The interval of the current statistics corresponds to the recent solar maximum period; thus, relatively many EPBs were observed in the low-altitude region in Ishigaki Island (magnetic latitude of Ishigaki is $19.6^{\circ} \mathrm{N}$ ). As will be introduced in a later part of the paper, we have started routine airglow measurements in Ishigaki with the lowcost ASI since December 2017 following the current pilot observation. However, the occurrence of EPBs is limited because of the significant decrease in solar activity during the present solar minimum.

Figure $4 \mathrm{~b}$ shows the same statistics of the occurrence of EPBs detected by the OMTI ASI in Ishigaki Island. Similar seasonal and local time dependences are identified. More importantly, most EPBs observed by the OMTI ASI are also detected by the low-cost ASI. The total number of nights, during which both the ASIs were operative and the OMTI ASI detected EPBs, is 32. The Watec ASI detected EPBs during 26 out of 32 nights of simultaneous observations. Hence, the Watec ASI was able to detect $\sim 81 \%$ EPBs that were seen in the OMTI ASI images. This result again confirms the capability of monitoring the appearance of EPBs using the low-cost ASI. We picked up EPBs from the Watec ASI by visual inspection without looking at the OMTI ASI images obtained at the same time. Hence, in some cases, we were not able to identify weak EPBs near the southern edge of the FOV.

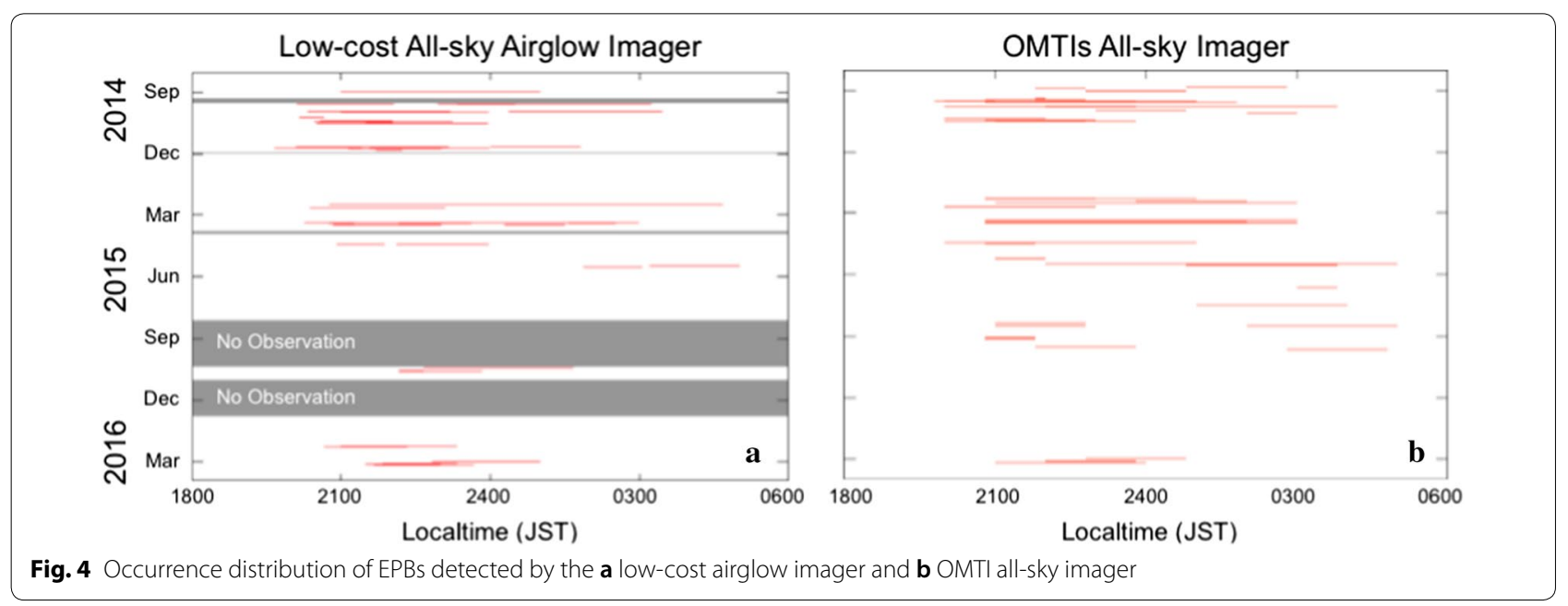


This is obviously because of the relatively lower $\mathrm{S} / \mathrm{N}$ ratio of the Watec ASI as compared to that of the OMTI ASI.

The other important point to note is that EPBs are detected only by the low-cost ASI, for example, those in November 2014. This is directly because the OMTI ASI did not operate in this interval, because the ASI did not operate when the Moon was above the horizon to prevent degradation of the CCD detector. In contrast to the OMTI ASI, the low-cost ASI has always been operated during the nighttime irrespective of the Moon phase. Of course, the low-cost ASI system, especially the CCD detector of the camera, is also affected by the strong illumination from the Moon. However, the camera can be replaced when the detector is significantly degraded, which enables us to use the low-cost ASI for monitoring EPBs all the time even during the full Moon period.

\section{Comparison to scintillation measurements}

As mentioned in the previous section, one of the significant space weather impacts of EPBs is their effect on GNSS scintillation. In Ishigaki Island, ENRI has been operating several GNSS receivers, whose sampling rate is $50 \mathrm{~Hz}$, to monitor the effect of the EPBs on the quality of navigation signals received on the ground. Figure 5 shows a comparison of the low-cost ASI data with the simultaneous scintillation measurements at 2337 JST on Feb 14,2015 . The deviation image is shown as a background image showing two EPB traces, one in the middle of the field-of-view and the other near the western horizon. The

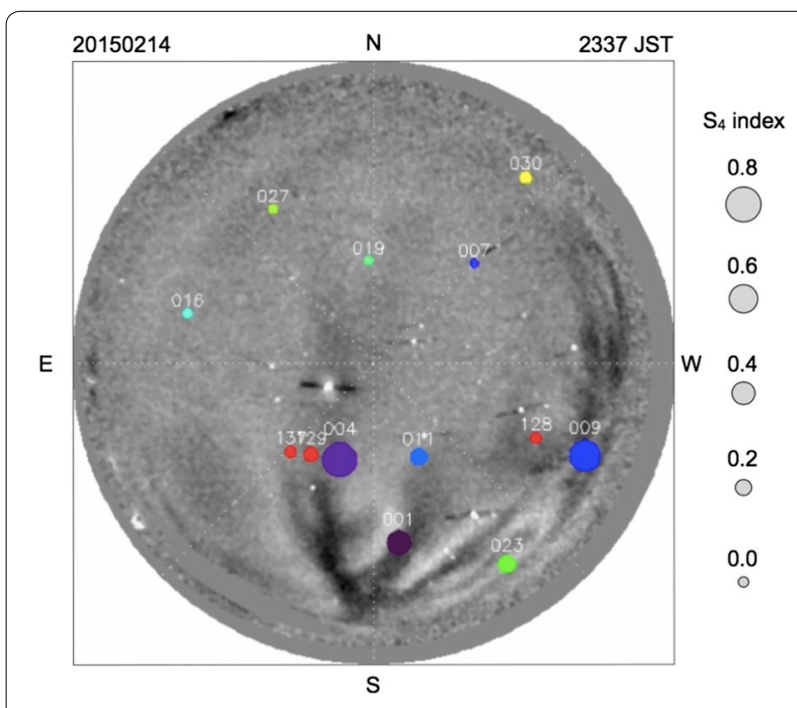

Fig. 5 Simultaneous observations of EPBs with the low-cost ASI and satellite scintillation measurements. The deviation image from the low-cost ASI is shown as a background. The circles give the location of the satellite within the all-sky field-of-view, and the size of the circles indicates the magnitude of the $\mathrm{S}_{4}$ index, which is a proxy for the amplitude scintillation circles show the direction of the satellites, including 10 GPS satellites and three geosynchronous satellites (reddish ones, 128, 129, and 137). The size of the circles indicates the magnitude of the $\mathrm{S}_{4}$ index, which is a proxy for the amplitude scintillation (e.g., Kintner et al. 2007). At this timing, the size of the circles is clearly larger in the southern half of the field-of-view where the ray-paths of several satellites cross the traces of EPBs. This tendency is clear for PRN1, PRN4, and PRN9, all of which are within the region of depleted airglow caused by the EPBs. This correspondence is consistent with previous observations that demonstrated the amplitude scintillation is directly associated with density irregularities embedded within EPBs (e.g., Kelley et al. 2002). Such a synchronizing behavior of the amplitude scintillation with the passage of EPBs is more clearly seen in an attached additional movie file covering the full sequence of the measurement on this night (see Additional file 2). In Fig. 5, it is found that the observed $\mathrm{S}_{4}$ values tend to be larger near the southern edge of the FOV. This could be because the length of the ray-path of the radio waves passing through EPBs is longer when the satellite is seen near the southern horizon. Such a viewing geometry also affects the level of scintillation as indexed by the magnitude of $\mathrm{S}_{4}$.

Figure 6 displays a time-series of the $\mathrm{S}_{4}$ index from the same interval, derived for the signals from each satellite. Figure $6 a-c$ shows the data for PRN 4, 7, and 11 of GPS satellites. Several prominent enhancements in the S4 index took place during the interval, the $\mathrm{S} 4$ index sometimes being higher than 1 . These green hatched intervals indicate periods of EPB passage detected by the lowcost imager. A one-to-one close relationship is clearly seen between the passages of EPBs and increases in the amplitude scintillation. This comparison implies that the optical data from the low-cost imager can be used to detect at least EPBs that can produce amplitude scintillation, which is of particular importance in monitoring the effect of EPBs on the GNSS.

Figure $6 \mathrm{~d}-\mathrm{f}$ shows the same data from three geosynchronous satellites (MTSAT-1, MTSAT-1R, and GSAT10). As the locations of these satellites do not move in the field-of-view of the ASI, the temporal variation of the $\mathrm{S}_{4}$ index during the full sequence of this EPB event can be studied. As is common in all the satellite data, the amplitude of the S4 index enhancement during the passages of the EPB was larger in the earlier timings, and the peak value decreased at later local time. This implies that the power of EPB-associated irregularities weakens during the course of the eastward propagation of EPBs. In the post-midnight part of Fig. 6f, for example, 02 to 05 JST, the $\mathrm{S}_{4}$ index did not increase even during the passage of EPBs. This suggests that the large-scale density depletions, such as EPBs, are maintained for a certain interval 

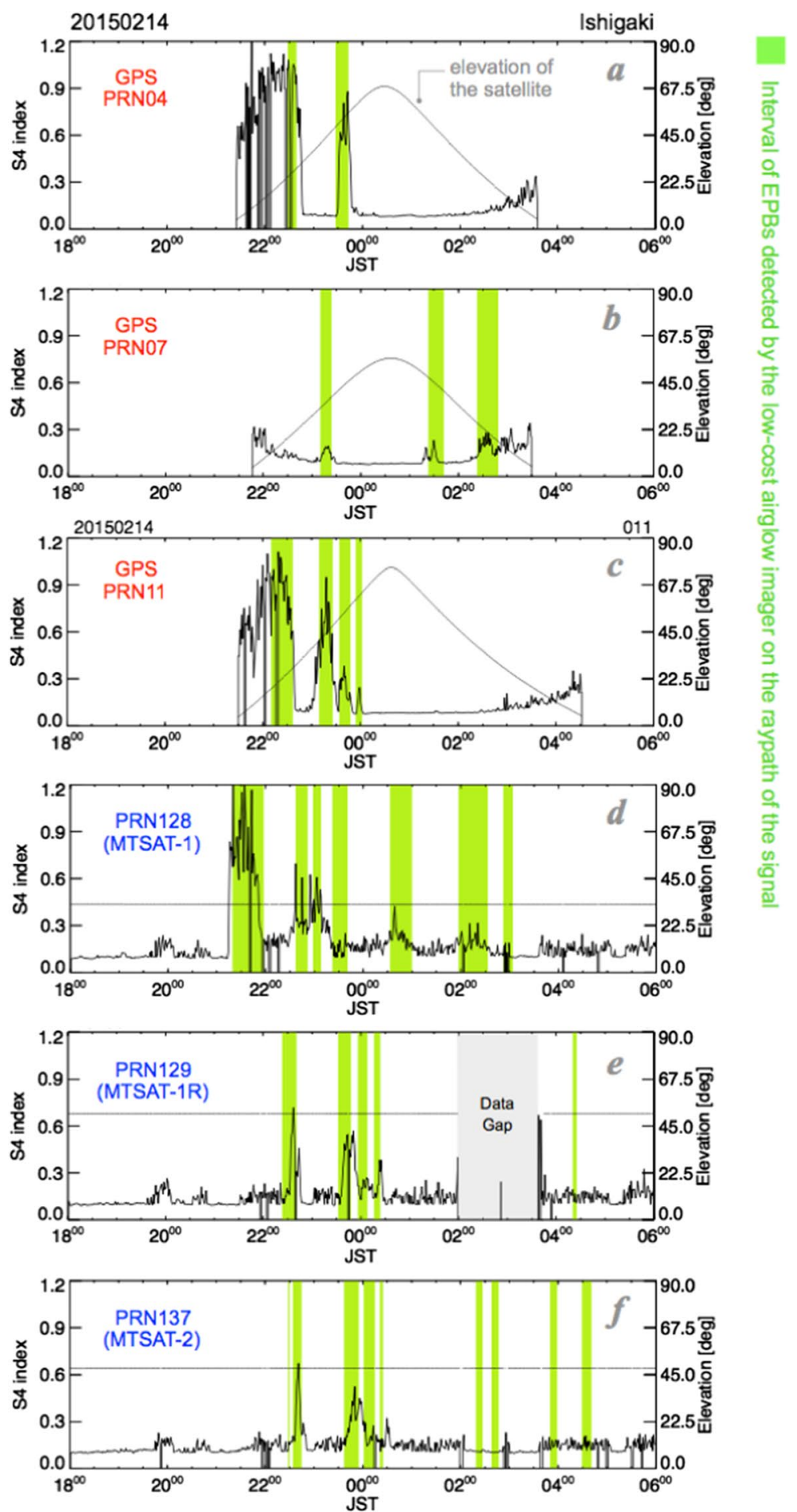

Fig. 6 Temporal variation of the $S_{4}$ index during the sequential passage of EPBs across Ishigaki Island on February 14, 2015. The data from three GPS satellites are shown in the top three panels $(\mathbf{a}-\mathbf{c})$ and those from three geosynchronous satellites are shown in the bottom three panels ( $\mathbf{d}-\mathbf{f}$ ). The intervals of EPBs detected by the low-cost airglow imager on the ray-path of the signal are highlighted green. The elevation angle of the satellite is overplotted 
after small-scale irregularities disappear (Basu et al. 1978).

\section{Monitoring of EPBs in a wide area using low-cost imager}

In the previous sections, we have demonstrated that the observations from the low-cost ASI could potentially detect EPBs. If the low-cost ASIs can be deployed in multiple places in the equatorial and low-latitude regions, it would be possible to visualize the large-scale distribution of EPBs and then monitor how they emerge and develop. To evaluate such a capability of large-scale monitoring of EPBs with multiple ASIs, we have conducted a pilot study to map the structure of EPBs by combining data of ASIs in Ishigaki and Tainan. Figure 7a shows the combined image of EPBs at 1355 UT on March 16, 2015. The imager in Tainan is not a low-cost ASI but one equipped with a cooled CCD camera. To reduce the oblique viewing effect, we plot the percentage deviation of the image. The oblique viewing effect equally affects the 2 -min average and 1-h average images; thus, the effect of oblique viewing should be reduced in the percentage deviation image.

On this night, several EPBs were observed traversing the field-of-view of the two ASIs from west to east, which is a typical eastward drift of EPBs. At the time of Fig. 7a, three traces of EPBs were identified in the combined image. Figure $7 \mathrm{~b}$ shows the corresponding keogram along the west-east cross-section at 20 geographic latitude (GLAT), indicating that the several EPBs drifted all the way across the fields-of-view of the two ASIs sequentially. The traces in the keogram are connected seamlessly at the boundary between the fields-of-view of the ASIs. This example demonstrates the capability of large-scale monitoring of EPBs with closely spaced multiple low-cost ASIs.

For the monitoring purpose of EPBs in a broad region, we have started routine observation of EPBs in Ishigaki Island following the feasibility study reported here. In addition to the low-cost ASI in Ishigaki, we also deployed the same low-cost ASIs in Ogimi, Okinawa $(26.68 \mathrm{~N}$, 128.15 E), Tainan, Taiwan (22.60 N, 120.10 E) and Chumphon, Thailand (10.72 N, $99.38 \mathrm{E})$ since early 2018. In the future, by expanding these network observations of the 630.0-nm airglow to a broad region covering East and Southeast Asia, we plan to set up a near-realtime monitoring system of EPBs in the equatorial and low-latitude regions in the Asian sector. For such monitoring observations, we may need to further improve the $\mathrm{S} / \mathrm{N}$ ratio of images from the low-cost ASIs. Now we are investigating a possibility to use a portable refrigerator as a housing of the ASI for reducing the random noise of instrumental origin. In addition, we are now testing an operation of

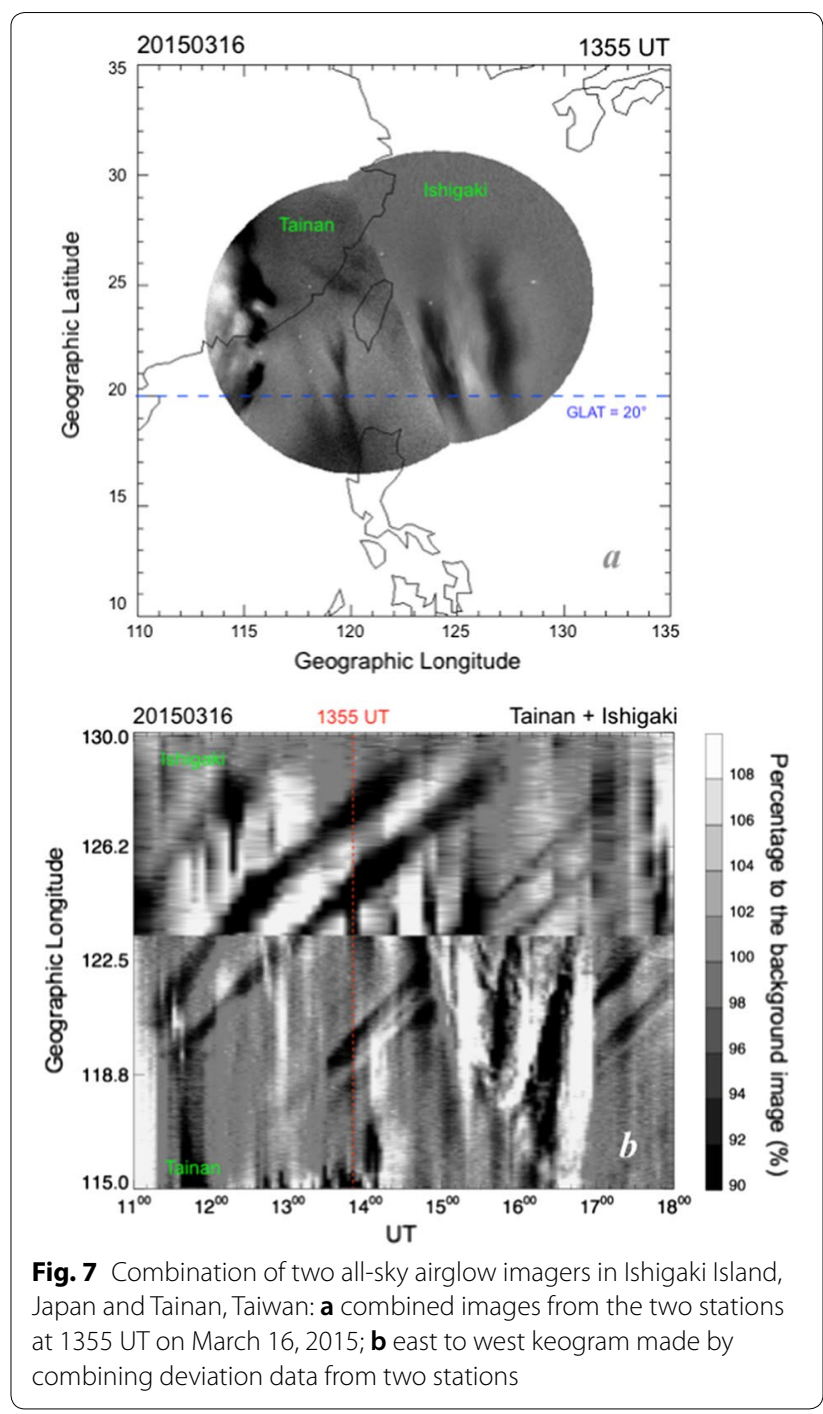

multiple cameras equipped with the same optical filters in the same place, which could improve the quality of observations by increasing the number of images for the integration process without blurring effect.

\section{Conclusion}

In this study, we evaluate the feasibility of observations of EPBs using a small and low-cost all-sky airglow imager. For this purpose, pilot observations using the low-cost imager system were conducted in Ishigaki Island in Japan over 1.5 years from 2014 to 2016 . This system consists of a small camera, fisheye lens, and optical filter, and it captures raw all-sky images every $4 \mathrm{~s}$. One of the problems of this low-cost ASI is the low $\mathrm{S} / \mathrm{N}$ ratio ( 0.5 in the raw images) due to shot noise from the non-cooled CCD camera. However, by averaging raw images for $2 \mathrm{~min}$ and extracting the 1-h average background image from the average image, 
we succeeded in visualizing the detailed spatial structure of plasma bubbles including the bifurcating traces whose horizontal scale size ranged from 30 to $100 \mathrm{~km}$. By comparing the L-band amplitude scintillation measurements with the low-cost ASI, we demonstrate that the level of amplitude scintillation proxied by the $S_{4}$ index enhanced significantly during the passage of plasma bubbles across the ray-path of the signal from the satellite. This further confirms that the low-cost ASI is able to monitor the impact of bubbles on the GNSS scintillation. Following the results of the current feasibility study of the low-cost ASI system, we plan to deploy the low-cost ASIs in low-latitude and equatorial Asian countries and establish a network observation for monitoring the generation and development of plasma bubbles and their space weather impacts on the satellite navigation system.

\section{Supplementary information}

Supplementary information accompanies this paper at https://doi. org/10.1186/s40623-020-01187-1.

Additional file 1: Movie S1. Full-sequence all-sky images obtained from the low-cost ASI during 2100 to 2400 JST on February 14, 2015. Left panel shows the raw image obtained with an exposure time of $4 \mathrm{~s}$. Right panel shows the average image obtained by integrating 30 consecutive images.

Additional file 2: Movie S2. Full-sequence all-sky deviation images from the low-cost ASI during $7 \mathrm{~h}$ interval from 2100 JST on February 14, 2015 to 0500 JST on February 15, 2015. The circles indicate the location of the satellite within the all-sky field-of-view, and the size of the circles indicates the magnitude of the $\mathrm{S}_{4}$ index, which is a proxy for the amplitude scintillation.

\section{Abbreviations}

CCD: Charge-coupled device; ASI: All-sky imager; EPBs: Equatorial plasma bubbles; GAIA: Ground-to-topside model of atmosphere and ionosphere for aeronomy; GNSS: Global navigation satellite system; GPS: Global positioning system; OMTIs: Optical mesosphere thermosphere imagers; FWHM: Full-width at half-maximum; ENRI: Electric Navigation Research Institute; LT: Local time; UT: Universal time; JST: Japan standard time; PRN: Pseudorandom noise; MTSAT: Multi-functional transport satellite; TIEGCM: Thermosphere-ionosphere electrodynamics general circulation model.

\section{Acknowledgements}

$\mathrm{KH}$ thanks the staff of the National Institute of Polar Research who operated and maintained the system for calibration of optical instruments. $\mathrm{KH}$ also thanks $\mathrm{H}$. Takayama for developing the low-cost airglow imager system deployed in Ishigaki Island.

\section{Authors' contributions}

$\mathrm{KH}$ designed and conducted the current research and prepared the manuscript. KT analyzed the optical and satellite scintillation data. SS operated the OMTI all-sky camera and satellite scintillation measurements in Ishigaki Island and helped the interpretation of the results. YAO developed and calibrated the low-cost all-sky imager used for the pilot observation. YO and KS operated and maintained the OMTI ASI in Ishigaki Island and discussed the results of the data analysis. All authors read and approved the final manuscript.

\section{Funding}

KH and SS are supported by JSPS-Kakenhi (17H02967). YUO and KS are supported by JSPS-Kakenhi (15H05815 and 16H06286).

\section{Availability of data and materials}

Data of low-cost airglow imager and satellite scintillation measurements from Ishigaki Island during the pilot measurement are available upon request (contact: Keisuke Hosokawa and Susumu Saito). The data of the OMTI ASI in Ishigaki are opened through ISEE, Nagoya University. Details can be found from the PWING Web site at https://www.isee.nagoya-u.ac.jp/dimr/PWING/ en. Digital data are available through the ERG Science Center of ISEE, Nagoya University (https://ergsc.isee.nagoya-u.ac.jp/index.shtml.en).

\section{Ethics approval and consent to participate}

Not applicable.

\section{Consent for publication}

Not applicable.

\section{Competing interests}

The authors declare that they have no competing interests.

\section{Author details}

${ }^{1}$ University of Electro-Communications, Chofugaoka 1-5-1, Chofu, Tokyo 182-8585, Japan. ${ }^{2}$ Electronic Navigation Research Institute, Jindaiji-Higashicho 7-42-23, Chofu, Tokyo 182-0012, Japan. ${ }^{3}$ Midoricho 10-3, Tachikawa, Tokyo 190-8518, Japan. ${ }^{4}$ Institute for Space-Earth Environmental Research, Nagoya University, Furocho, Chikusa-ku, Nagoya, Aichi 464-8601, Japan. ${ }^{5}$ Department of Earth Sciences, National Cheng Kung University, Tainan, Taiwan.

Received: 13 November 2019 Accepted: 25 April 2020

Published online: 06 May 2020

\section{References}

Aarons J (1993) The longitudinal morphology of equatorial F-layer irregularities relevant to their occurrence. Space Sci Rev 63:209-243

Basu S, Basu S, Aarons J, McClure JP, Cousins MD (1978) On the coexistence of kilometer-and meter-scale irregularities in the nighttime equatorial $F$ region. J Geophys Res 83:4219-4226. https://doi.org/10.1029/JA083iA09p 04219

Carter BA et al (2014) Geomagnetic control of equatorial plasma bubble activity modeled by the TIEGCM with Kp. Geophys Res Lett 41:5331-5339. https://doi.org/10.1002/2014GL060953

Fejer BG, Kelley MC (1980) Ionospheric irregularities. Rev Geophys 18:401-454. https://doi.org/10.1029/RG018i002p00401

Franke SJ, Liu CH, McClure JP (1984) Interpretation and modeling of quasiperiodic diffraction patterns observed in equatorial VHF scintillation due to plasma bubbles. J Geophys Res 89:10891-10902. https://doi.org/10.1029/ JA089iA12p10891

Hosokawa K, Ogawa Y, Taguchi S (2019) Imaging of polar cap patches with a low-cost airglow camera: pilot observations in Svalbard, Norway. Earth Planets Space 71:115. https://doi.org/10.1186/s40623-019-1094-7

Huang C-S, Kelley MC (1996) Nonlinear evolution of equatorial spread F: 2 gravity wave seeding of Rayleigh-Taylor instability. J Geophys Res 101:293-302

Kelley MC, Makela JJ, Ledvina BM, Kintner PM (2002) Observations of equatorial spread-F from Haleakala, Hawaii. Geophys Res Lett 29:64. https://doi. org/10.1029/2002GL015509

Kil H (2015) The morphology of equatorial plasma bubbles_a review. J Astron Space Sci 32:13-19

Kil H, Paxton LJ, Oh S-J (2009) Global bubble distribution seen from ROCSAT-1 and its association with the pre-reversal enhancement. J Geophys Res 114:A06307. https://doi.org/10.1029/2008JA013672

Kintner PM, Ledvina BM, de Paula ER (2007) GPS and ionospheric scintillations. Space Weather 5:S09003. https://doi.org/10.1029/2006SW000260

Makela JJ, Ledvina BM, Kelley MC, Kintner PM (2004) Analysis of the seasonal variations of equatorial plasma bubble occurrence observed from Haleakala, Hawaii. Ann Geophys 22:3109-3121. https://doi.org/10.5194/ angeo-22-3109-2004

Maruyama T, Matuura N (1984) Longitudinal variability of annual changes in activity of equatorial spread F and plasma bubbles. J Geophys Res 89:10903-10912 
Ogawa Y, Tanaka Y, Kadokura A, Hosokawa K, Ebihara Y, Motoba T, Gustavsson B, Brändström U, Sato Y, Oyama S, Raita T, Sigernes F, Nozawa S, Shiokawa K, Kosch M, Kauristie K, Hall C, Suzuki S, Miyoshi Y, Gerrard A, Miyaoka H, Fujii R (2020) Development of low-cost multi-wavelength imager system for studies of auroras and airglows. Polar Sci. https://doi.org/10.1016/j. polar.2019.100501

Otsuka Y, Shiokawa K, Ogawa T, Wilkinson P (2002) Geomagnetic conjugate observations of equatorial airglow depletions. Geophys Res Lett 29:43. https://doi.org/10.1029/2002GL015347

Shinagawa H, Jin H, Miyoshi Y, Fujiwara H, Yokoyama T, Otsuka Y (2018) Daily and seasonal variations in the linear growth rate of the Rayleigh-Taylor instability in the ionosphere obtained with GAIA. Prog Earth Planet Sci $5: 16$

Shiokawa K, Katoh Y, Satoh M, Ejiri MK, Ogawa T, Nakamura T, Tsuda T, Wiens $\mathrm{RH}$ (1999) Development of optical mesosphere thermosphere imagers (OMTI). Earth Planets Space 51:887-896. https://doi.org/10.1186/BF033 53247

Shiokawa K, Otsuka Y, Ogawa T, Wilkinson P (2004) Time evolution of highaltitude plasma bubbles imaged at geomagnetic conjugate points. Ann Geophys 22:3137-3143

Shiokawa K, Otsuka Y, Ogawa T (2009) Propagation characteristics of nighttime mesospheric and thermospheric waves observed by optical mesosphere thermosphere imagers at middle and low latitudes. Earth Planets Space 61:479-491. https://doi.org/10.1186/BF03353165

Sobral JHA, Takahashi H, Abdu MA, Muralikrishna P, Sahai Y, Zamlutti CJ, de Paula ER, Batista PP (1993) Determination of the quenching rate of the O(1D) by O(3P) from rocket-borne optical $(630 \mathrm{~nm})$ and electron density data. J Geophys Res 98(A5):7791-7798. https://doi.org/10.1029/92JA0 1839

Tsunoda RT (2015) Upwelling: a unit of disturbance in equatorial spread F. Prog Earth Planet Sci 2:9. https://doi.org/10.1186/s40645-015-0038-5

Weber EJ, Buchau J, Eather H, Mende SB (1978) North-south aligned equatorial airglow depletions. J Geophys Res 83:712-716

Woodman RF, Lahoz C (1976) Radar observations of F region equatorial irregularities. J Geophys Res 81:5447-5466

Yokoyama T (2017) A review on the numerical simulation of equatorial plasma bubbles toward scintillation evaluation and forecasting. Prog Earth Planet Sci 4:37. https://doi.org/10.1186/s40645-017-0153-6

\section{Publisher's Note}

Springer Nature remains neutral with regard to jurisdictional claims in published maps and institutional affiliations.

\section{Submit your manuscript to a SpringerOpen ${ }^{\circ}$ journal and benefit from:}

- Convenient online submission

- Rigorous peer review

- Open access: articles freely available online

- High visibility within the field

Retaining the copyright to your article

Submit your next manuscript at $\boldsymbol{\nabla}$ springeropen.com 